Check for updates

Cite this: RSC Adv., 2018, 8, 31502

Received 22nd July 2018

Accepted 27th August 2018

DOI: $10.1039 / c 8 r a 06196 c$

rsc.li/rsc-advances

\section{Designing an efficient graphene quantum dot-filled luminescent down shifting layer to improve the stability and efficiency of perovskite solar cells by simple optical modeling $\dagger$}

\begin{abstract}
Zahra Hosseini iD * and Teymoor Ghanbari
Degradation of perovskite material under UV light is a problem hampering the practical application of perovskite solar cells (PSCs) despite attaining high efficiency. This paper studies the application of a luminescent down shifting (LDS) layer containing graphene quantum dots (GQDs) on top of a PSC as an efficient strategy to improve the stability and light harvesting efficiency of PSCs under UV light. With absorption and emission bands in the UV and visible regions respectively, and simple synthesis of GQDs with a high luminescence quantum efficiency (QE), GQDs are a suitable candidate as a down shifting material in the LDS layer. Here, a simple optical model is used to investigate the effect of parameters such as the concentration of GQDs, LDS layer thickness, absorption/emission bands of GQDs and the luminescence quantum efficiency on the performance of the LDS layer. The calculated results show that application of a GQD-filled LDS layer, with 94\% QE and negligible RO and PA, on a PSC causes a remarkable enhancement in the incident photon to current conversion efficiency (IPCE) and thereby the short circuit current density $\left(J_{S C}\right)$ in the $300-400 \mathrm{~nm}$ spectral range of more than $400 \%$. This strategy is also very effective in improving the stability of the PSC by suppressing the UV light from entering the device.
\end{abstract}

\section{Introduction}

Perovskite solar cells (PSCs) have garnered much attention over the past few years owing to the simplicity of fabrication and rapid advancement of their power conversion efficiency (PCE). ${ }^{1-4}$ To date, PSCs have achieved PCEs of over $22 \% .^{5}$ The organometallic halide perovskite which is the light harvester in the PSCs exhibits broad light absorption, a large extinction coefficient, and high carrier mobility. ${ }^{\mathbf{1}, 2}$ Despite the high PCEs, organic-inorganic perovskites suffer from a number of instability mechanisms; this is the main obstacle on the path of PSCs toward commercial viability. Degradation of organicinorganic perovskites has been observed mainly in the presence of moisture, at elevated temperatures and under UV irradiation. ${ }^{6-13}$ Several approaches have been proposed to overcome the instabilities of PSC $^{9}$ and significant progress has been made in this regard. Nevertheless, PSCs still severely suffer from inadequate photo-stability under continuous solar radiation especially UV radiation. The high energy UV photons have been recognized to deteriorate the perovskite

Faculty of Advanced Technologies, Shiraz University, Shiraz, Iran, PO Box: 7194684560. E-mail: zahrahosseini@shirazu.ac.ir

$\dagger$ Electronic supplementary information (ESI) available. See DOI: 10.1039/c8ra06196c materials. ${ }^{\mathbf{8 , 1 4}}$ Thus, protection of PSCs from UV light seems to be crucial.

One promising strategy to address this problem is application of a luminescent down shifting (LDS) layer on top of a PSC. The LDS layer helps hinder the UV photons from reaching the perovskite material while these photons still contribute in current generation. This layer contains a luminescent material that absorbs the short-wavelength photons of the solar spectrum - which are not used efficiently by the solar cell-and then re-emits them at longer wavelengths. The long wavelength, downshifted photons are absorbed and converted into electricity by the underlying solar cell more efficiently, therefore improving its current generation and the overall PCE. ${ }^{15}$ This approach has been developed to overcome different stability and spectral mismatch losses by different spectral conversion processes such as down shifting, down conversion and up conversion in various photovoltaic (PV) devices. ${ }^{16-20}$ Conversion of UV light to visible photons by luminescent down shifting materials has also been used to overcome the degradation of perovskite material under irradiation of UV light. ${ }^{7,8,21,22}$ A careful study of the corresponding reports shows that although a better stability has been achieved in all efforts, the amount of light harvesting efficiency is not considerable in UV region. Therefore, not a remarkable enhancement in PCE is attained. This is attributed to the 
luminescent down shifting material embedded in the LDS layer. A luminescent down shifting material using in this strategy must satisfy specific requirements to maximize the benefits from the application of the LDS layer as follows. The appropriate material possesses a wide absorption band in UV region and exhibits a luminescence spectrum that matches the absorption spectrum of the perovskite material in PSC. To avoid loss due to re-absorption of photons emitted by luminescent species, the overlap between the emission spectrum and the excitation spectrum of the luminescent material should be minimal. The luminescent material should also possess a high luminescence quantum efficiency (QE), large absorption coefficient, adequate photochemical stability and low price. ${ }^{15,23}$ Graphene quantum dots (GQDs) are zerodimensional materials with average sizes less than $10 \mathrm{~nm}$ that have size-tunable bandgaps up to $3 \mathrm{eV}$. They have exhibited novel light absorption/emission phenomena. ${ }^{24-26}$ Depending on the synthesis method, precursors and doping, they may show excitation wavelength dependent/independent emission with different degrees of overlap between the absorption and emission bands..$^{25,27-30}$ They usually absorb in UV region and emit visible photons. Moreover, compared to conventional quantum dots (QDs), GQDs have some particularly unusual chemical/physical properties, such as low toxicity, chemical inertness, solubility, functionality, crystallinity, two-dimensionality, biocompatibility, and stable photoluminescence. ${ }^{31,32}$ Owing these properties, GQDs seems to be proper candidates for application in the LDS layers.

In this paper, we explore the feasibility of GQD use in LDS layer on top of PSCs. An optical model is used to assess the effects of the incorporation of GQDs in a planar plastic layer on top of the PSC. The assessment includes different absorption/ emission spectra and concentrations of GQD, and LDS layer thicknesses. We also study the effects of luminescent QE on the performance of the LDS layer and will discuss the optimum conditions.

\section{Optical modeling}

Fig. 1 illustrates the concept of an LDS layer coupled with a PSC. Luminescent GQDs are dispersed in a transparent plastic layer to make the LDS layer. The LDS layer then is put on top of the PSC. The optical processes involved in this configuration are shown by arrows. A fraction of incident photons is reflected at the air/LDS layer interface. Another fraction with a wavelength in the absorption band of the perovskite material are transmitted from the LDS layer and are absorbed by perovskite layer inside the PSC. The high-energy photons are absorbed by GQDs inside the LDS layer. The GQDs then re-emit the absorbed photons at lower energies. The emitted photons go back to the PSC to be absorbed by perovskite material either directly or after reflection from the LDS layer/air interface. The emitted photons may be re-absorbed by other GQDs in the LDS layer if there is an overlap between the absorption and emission bands of the GQD. A fraction of photons emitted by the GQDs escape out of the LDS layer through the LDS layer/air interface or from the side planes.

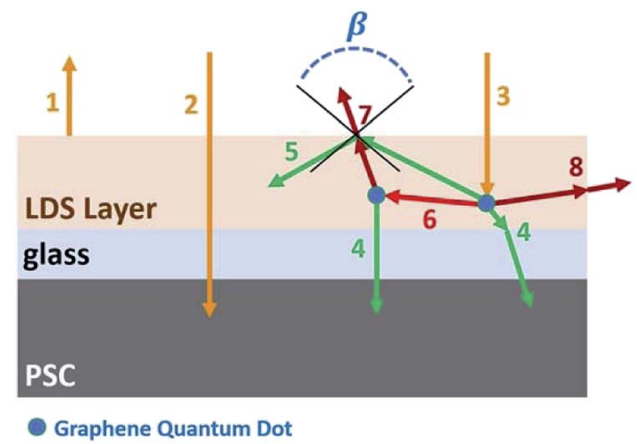

Fig. 1 Schematic of optical processes involved in the LDS layer on top of a PSC. The incident photons will be partly reflected from the device (1), partly transmit and enter the PSC where they can be absorbed by the perovskite material (2) while the rest are absorbed in the LDS layer (3). The GQDs then re-emits the absorbed photons at lower energies. The emitted photons illuminate the solar cell either directly (4) or after reflection from the LDS layer/air interface (5). A part of the emitted photons is re-absorbed by other GQDs present in the LDS layer (6) and a fraction of them escapes from the front surface of the LDS layer/air interface (7) or edge planes (8)

As the incoming photons (air mass 1.5 global (AM1.5G) solar radiation) reach the PSC after crossing the LDS layer in this configuration, the total incident photon to current conversion efficiency (IPCE) of PSC with LDS layer comprises the current generated by photons transmitted from the LDS layer and reach the PSC directly, and the current generated by the down shifted photons in the LDS layer. Therefore, the IPCE of the PSC with an LDS layer can be simply calculated as:

$$
\mathrm{IPCE}=\mathrm{IPCE}_{\mathrm{d}}+\mathrm{IPCE}_{\mathrm{LDS}}
$$

in which the $\operatorname{IPCE}_{\mathrm{d}}$ is the IPCE associated with the photons that reach the PSC directly after passing through the LDS layer and IPCE $_{\mathrm{LDS}}$ is the IPCE generated by the down shifted photons which reach the PSC. Since the photons that reach the PSC directly or after wavelength shifting are a fraction of the incident AM1.5G solar spectrum, IPCE $\mathrm{d}_{\mathrm{d}}$ and $\mathrm{IPCE}_{\mathrm{LDS}}$ are individually less than the IPCE of a bare PSC device whereas the sum of them can adopt a greater value than the IPCE of the bare device. Also, taking into account that the ideal luminescent QE is $100 \%$, this will ensure that (1) will never have a quantity greater than unity.

The distribution of transmitted photons from the LDS layer $\left(S_{\mathrm{T}}(\lambda)\right)$ which reach inside the PSC (arrow 2 in Fig. 1) can be calculated by using the initial solar spectrum $\left(S_{\mathrm{i}}(\lambda)\right)$, transmission spectrum of the LDS layer $\left(T_{\mathrm{LDS}}(\lambda)\right)$, and reflection spectrum of PSC device with the LDS layer on top $\left(R_{\text {device }}(\lambda)\right)$ :

$$
S_{\mathrm{T}}(\lambda)=S_{\mathrm{i}}(\lambda) T_{\mathrm{LDS}}(\lambda)\left(1-R_{\text {device }}(\lambda)\right)
$$

The LDS layer is a plastic layer containing GQDs. Therefore, the transparency of this layer is affected by both the host material and the GQDs, which means:

$$
T_{\mathrm{LDS}}(\lambda)=T_{\mathrm{H}}(\lambda) T_{\mathrm{GQD}}(\lambda)
$$


in which $T_{\mathrm{H}}$ and $T_{\mathrm{GQD}}$ are the host and GQD transmission, respectively.

Then, the $\mathrm{IPCE}_{\mathrm{d}}$ can be calculated as follows:

$$
\operatorname{IPCE}_{\mathrm{d}}(\lambda)=T_{\mathrm{LDS}}(\lambda) \frac{1-R_{\text {device }}}{1-R_{\text {bare }}} \operatorname{IPCE}_{\text {bare }}(\lambda)
$$

in which $R_{\text {bare }}$ and IPCE $_{\text {bare }}$ are the reflection and IPCE of the bare PSC.

In calculation of the $R_{\text {device }}(\lambda)$, two interfaces should be taken into account: air/LDS layer interface and LDS layer/glass interface with reflections $R_{1}$ and $R_{2}$ respectively. For normal incidence of photons, the reflection from these two interfaces $\left(R_{1} \&\right.$ $R_{2}$ ) can be calculated from the Fresnel equations:

$$
\begin{gathered}
R_{1}=\left(\frac{1-n_{\mathrm{LDS}}}{1-n_{\mathrm{LDS}}}\right)^{2} \\
R_{2}=\left(\frac{n_{\mathrm{LDS}}-n_{\text {glass }}}{n_{\mathrm{LDS}}+n_{\text {glass }}}\right)^{2}
\end{gathered}
$$

in which $n_{\text {LDS }}$ and $n_{\text {glass }}$ are the refractive indices of the LDS layer and glass substrate, respectively. Considering the multiple specular reflections within the LDS layer for normal incident photons, the $R_{\text {device }}(\lambda)$ can be calculated as: ${ }^{33}$

$$
R_{\text {device }}(\lambda)=\frac{R_{1}+\left(1-R_{1}^{2}\right) T_{\mathrm{LDS}^{2}(\lambda) R_{2}}}{1-R_{1} T_{\mathrm{LDS}}{ }^{2}(\lambda) R_{2}}
$$

The rest of the incident photons, which are not transmitted or reflected from the device, are absorbed in the LDS layer (arrow 3 in Fig. 1). Then the LDS layer re-emits these photons at longer wavelengths. The number of emitted photons is affected by two factors. The first one is the ratio of the absorbed photons by GQDs to the total absorbed photons by LDS layer $\left(\frac{\log \left[T_{\mathrm{GQD}}(\lambda)\right]}{\log \left[T_{\mathrm{LDS}}(\lambda)\right]}\right)$. This factor should be included because out of all photons absorbed in LDS layer, only the photons absorbed by GQDs can be down shifted to lower energy photons. The second factor is the GQDs luminescent quantum efficiency which determines the ratio of emitted photons to the absorbed photons by GQDs.

On the other hand, considering a homogeneous angular distribution for emitted photons, not all the emitted photons in the LDS layer reach the PSC. A fraction of photons which emits into the angles less than the critical angle at the LDS layer/air interface escapes from the LDS layer (arrow 7 in Fig. 1). The probability of the down shifted photons being emitted within the escape cone $(\beta)$ is given by: ${ }^{34}$

$$
\beta=\frac{1}{2}\left(1-\left(1-\frac{1}{n_{\mathrm{LDS}}^{2}}\right)^{\frac{1}{2}}\right)
$$

The other fraction of photons emitted outside the escape cone has a second chance to reach the PSC (arrow 5 in Fig. 1). Depending on the thickness of the LDS layer, there is a small probability $(\alpha)$ that the emitted photons escape from the side planes of the LDS layer (arrow 8 in Fig. 1). Moreover, there is a probability (RO) that the down shifted photons being reabsorbed by another GQD in the LDS layer (arrow 6 in Fig. 1). Considering all these processes, a collection probability can be approximated by: ${ }^{33}$

$$
P=\frac{(1-\alpha)\left(1-\mathrm{RO}_{\mathrm{O}}\right)(1-\beta)}{1-\mathrm{QE}\left[\mathrm{RO}_{\mathrm{I}} \beta+(1-\beta) \mathrm{RO}_{\mathrm{O}}\right]}
$$

in which the $\mathrm{RO}_{\mathrm{O}}$ and $\mathrm{RO}_{\mathrm{I}}$ determines the probability of reabsorption of photons emitted outside and inside the escape cone, respectively. Re-absorption probability is calculated by: ${ }^{35}$

$$
\mathrm{RO}=\frac{\int \operatorname{PL}(\lambda)\left[1-10^{-d C \varepsilon(\lambda)}\right] \mathrm{d} \lambda}{\int \operatorname{PL}(\lambda) \mathrm{d} \lambda}
$$

where $\operatorname{PL}(\lambda)$ is the emission spectrum of GQDs, $C$ is the GQD concentration, $\varepsilon(\lambda)$ is the GQD extinction coefficient and $d$ is chosen to be $d=D / 2$ and $d=3 D^{33}$ for calculating $\mathrm{RO}_{\mathrm{I}}$ and $\mathrm{RO}_{\mathrm{O}}$, respectively. $D$ is the LDS layer thickness.

The IPCE associated with the down shifted photons then is calculated as:

$$
\begin{aligned}
& \operatorname{IPCE}_{\mathrm{LDS}}(\lambda)= \\
& \quad\left(1-R_{\text {device }}(\lambda)\right)\left(1-T_{\mathrm{LDS}}(\lambda)\right) \frac{\log \left[T_{\mathrm{GQD}}(\lambda)\right]}{\log \left[T_{\mathrm{LDS}}(\lambda)\right]} P \cdot \mathrm{QE} \cdot \mathrm{ESM}
\end{aligned}
$$

in which ESM is the emission spectral matching factor. So that the down shifted photons contribute to current generation, the emission spectrum of the GQD must effectively overlap the IPCE of the bare PSC device; these wavelengths then provide a large IPCE $_{\mathrm{LDS}}$. The ESM is thus defined as the fraction of the emission that overlaps the IPCE $\mathrm{bare}_{\text {: }}$

$$
\operatorname{ESM}=\frac{\int \operatorname{PL}(\lambda) \operatorname{IPCE}_{\text {bare }}(\lambda) \mathrm{d} \lambda}{\int \operatorname{PL}(\lambda) \mathrm{d} \lambda}
$$

\section{Results and discussions}

The optical model described above is used to calculate the IPCE of PSC coupled with an LDS layer containing GQDs. The effect of different parameters on the operation of the LDS layer is investigated and the optimal parameters are suggested. These parameters are concentration of GQDs in the LDS layer, LDS layer thickness, GQD absorption/emission bands with different overlaps and luminescent QE of GQD. Although all GQDs absorb UV light and emit visible photons, depending on their size, shape, doping, functional groups and synthesis method, their absorption/emission bands are slightly different with different QEs. In addition, GQDs sometimes show excitationdependent emission. ${ }^{27,36}$

The GQD related input for the optical model is adapted from ref. 25. In ref. 25 the synthesis and photoluminescence behavior of a series of N-doped GQDs are reported. The absorption/ emission spectra of GQD-EDA and GQD-G with 94\% and 6\% QEs are used here. GQD-EDA was synthesized under hydrothermal processes using citric acid (CA) and ethylene diamine (EDA) as the carbon precursor and nitrogen dopant 


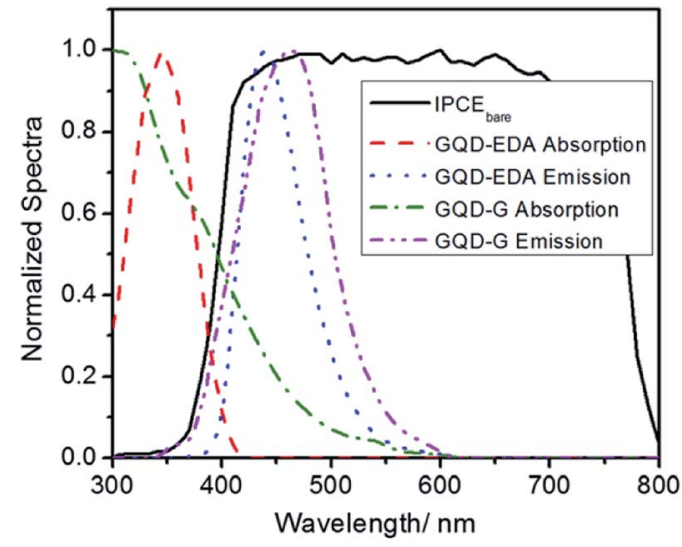

Fig. 2 Normalized absorption and emission spectra of GQD-EDA and GQD-G compared to the IPCE of a bare PSC. ${ }^{21,25}$

respectively. ${ }^{25}$ GQD-G was synthesized using glucose as carbon source. ${ }^{25}$ Fig. 2 shows the absorption/emission spectra of these GQDs together with a typical IPCE of a PSC. The IPCE of PSC is adapted from ref. 21. For both GQDs, there is a good overlap between the emission band of the GQD and the IPCE of PSC; this provides large ESM factor. Further, both GQDs absorb in UV region. There are several fundamental differences between the two kinds of GQD investigated in this research. The differences are the QEs and the amounts of RO between their absorption/emission bands. Also, the absorption bands of these GQDs have different degrees of overlap with the IPCE of PSC, i.e., parasitic absorption (PA). Therefore, the investigation of the effect of RO and PA is also possible.

Poly(methyl methacrylate) (PMMA) is a popular host for making LDS layer because its transparent in UV/Vis region and have refractive index of 1.489 . Therefore, there will be no loss due to the light absorption by host material and the reflection at air/LDS layer interface is negligible. We also used PMMA characteristics as the host input in the calculations.

\section{Effect of GQD concentration and LDS layer thickness}

In down shifting strategy, the first step is the absorption of UV light by LDS layer. In an efficient LDS layer, almost all UV photons are absorbed by the luminescent species. Light absorption by LDS layer increases by increasing both GQDs concentration and the LDS layer thickness. Enhanced light absorption by GQDs in the LDS layer leads to an increased number of down shifted photons and thereby an increase in the IPCE in short wavelength region. Increasing the LDS layer thickness not only increases the light absorption by GQDs but also increases the light absorption by host material. Light absorption by host material is a loss channel because the photons absorbed by the host material do not contribute in current generation in the solar cell. Therefore, one should use as thin as possible LDS layer in order to minimize the light absorption by host material. However, a smart choice of host material which is transparent in UV/Visible region will let us to use a thick LDS layer. Even in this case, one should notice that in a thick LDS layer, there is a higher chance for down shifted photons to escape from edge planes and thereby make the edge loss $(\alpha)$ notable. Therefore, thin LDS layers are preferable. However, there are also other factors affecting the choice of the thickness of the LDS layer. When the down shifting luminescent material has a low extinction coefficient, thin LDS layers cannot be used because the light absorption by luminescent particles will not be enough to make an efficient down shifting effect. Also, agglomeration of particles may happen if they used in high concentration in a thin LDS layer and the QE will thus decrease. Having high extinction coefficient around $10^{5} \mathrm{M}^{-1} \mathrm{~cm}^{-1}$, the GQDs can be effective even when using in low concentrations or in thin LDS layer. Therefore, we investigated the effect of GQD concentration in an LDS layer with $D=1 \mu \mathrm{m}$. However, the result of IPCE calculation for different thicknesses of LDS layer is provided in Fig. S1 in ESI. $\dagger$

Calculated IPCEs of PSCs with LDS layers containing different GQD-EDA concentrations in the range of $10 \mu \mathrm{M}$ to $1 \mathrm{M}$ is reported in Fig. 3a. It is supposed that edge loss $(\alpha)$ is negligible because the LDS layer is thin. One observes that application of the LDS layer containing GQD-EDA on PSC leads to an enhancement in IPCE in spectral range 300-400 nm coinciding with the absorption band of the GQD-EDA shown in Fig. 2. The enhanced IPCE in 300-400 $\mathrm{nm}$ spectral range which is caused by the down shifting of photons absorbed by GQDs in this range, is greatly affected by the concentration of these particles in the LDS layer. At wavelength less than $400 \mathrm{~nm}$, the IPCE
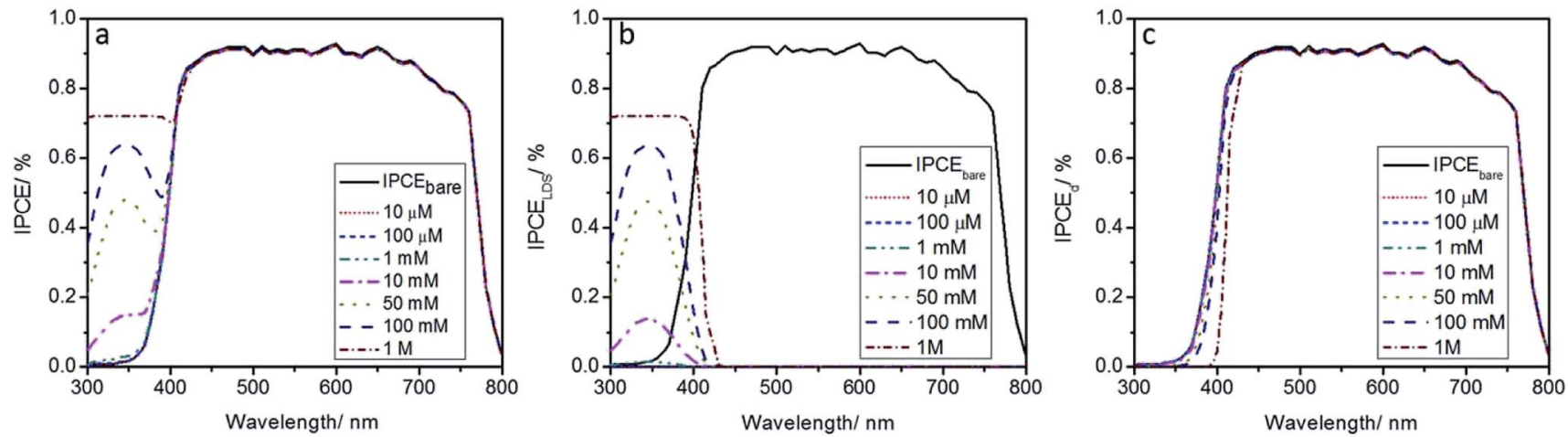

Fig. 3 Calculated (a) total IPCE spectra, (b) IPCE $E_{L D S}$ and (c) IPCE $E_{d}$ for PSCs without and with LDS layers containing GQD-EDA with different concentrations. 
values of the devices coupled with LDS layer are significantly greater than that of the bare device when using GQD-EDA in concentrations greater than $10 \mathrm{mM}$. IPCE attains $72 \%$ near $300 \mathrm{~nm}$ for the device with LDS layer containing $1 \mathrm{M}$ GQD-EDA; this value cannot exceed $10 \%$ in a conventional bare PSC. The reason for the IPCE enhancement is readily understood from Fig. $3 b$ and $c$ which show the calculated IPCE $E_{L D S}$ and IPCE $_{d}$ for the same devices respectively. IPCE $_{\mathrm{LDS}}$ is the portion of IPCE corresponding to the down shifting effect whereas $\mathrm{IPCE}_{\mathrm{d}}$ corresponds to the photons which are absorbed by the PSC directly. By increasing the concentration of GQD-EDA particles in the LDS layer, the IPCE $E_{\mathrm{LDS}}$ increases (Fig. 3b) and $\operatorname{IPCE}_{\mathrm{d}}$ is almost unchanged (Fig. 3c). The enhancement in $\mathrm{IPCE}_{\mathrm{LDS}}$ is significant especially when the concentration is greater than $10 \mathrm{mM}$. This is attributed to the larger number of absorbed and thereby down shifted photons in the LDS layer when higher concentration of GQD-EDA particles embed in this layer. In Fig. 3c, a slight decrease in calculated $\mathrm{IPCE}_{\mathrm{d}}$ is observed for $1 \mathrm{M}$ concentration of GQD-EDA which is due to the small overlap between the absorption band of GQD-EDA and $\mathrm{IPCE}_{\mathrm{bare}}$, i.e., PA. More discussion on PA is provided in the next section.

The effect of the down shifting strategy on the short-circuit current density $J_{\mathrm{SC}}$ of the PSC is realized from Table 1 , which shows the percentage increase in $J_{\mathrm{SC}}\left(\Delta J_{\mathrm{SC}} / \%\right)$ as $\left(J_{\mathrm{SC}, \mathrm{LDS}}-\right.$ $\left.J_{\mathrm{SC}, \text { bare }}\right) / J_{\mathrm{SC}, \text { bare }} ; J_{\mathrm{SC}, \mathrm{LDS}}$ and $J_{\mathrm{SC} \text {,bare }}$ represent the short-circuit current densities of devices with and without an LDS layer, respectively. The short-circuit current densities of the PSCs are calculated from the corresponding IPCE spectra shown in Fig. 3a. Table 1 shows this value for 300-400 $\mathrm{nm}$ and 400$800 \mathrm{~nm}$ spectral range separately. The current density generated due to the absorption of photons in 400-800 nm spectral range do not change by application of LDS layer containing GQD-EDA whereas a remarkable increase in current density generated in 300-400 nm spectral range is achieved by application of this layer on PSC. This is attributed to the down shifting range which coincides with the absorption band of GQD-EDA (i.e., 300-400 $\mathrm{nm})$. In 400-800 $\mathrm{nm}$ spectral range, the GQD-EDA particles do not absorb and application of LDS layer do not change the $J_{\mathrm{SC}}$ in this range notably. The small negative change in $J_{\mathrm{SC}}$ in $400-$ $800 \mathrm{~nm}$ spectral range is due to the device reflection or light absorption by the host material in LDS layer which decrease the

Table 1 Relative increase/\% in short-circuit current density of the PSCs with LDS layers containing different concentrations of GQD-EDA with respect to that of the reference cell for two wavelength ranges. The quantities are calculated from the corresponding IPCE spectra. The thickness of the LDS layers is $1 \mu \mathrm{m}$

\begin{tabular}{lcl}
\hline & $\Delta J_{\mathrm{SC}} / \%$ & \\
\cline { 2 - 3 } GQD-EDA concentration & $300-400 \mathrm{~nm}$ & $400-800 \mathrm{~nm}$ \\
\hline $10 \mu \mathrm{M}$ & -0.6 & -0.5 \\
$100 \mu \mathrm{M}$ & 0.0 & -0.5 \\
$1 \mathrm{mM}$ & 5.7 & -0.5 \\
$10 \mathrm{mM}$ & 5.8 & -0.5 \\
$50 \mathrm{mM}$ & 215.2 & -0.5 \\
$100 \mathrm{mM}$ & 311.3 & -0.5 \\
$1 \mathrm{M}$ & 414.7 & -0.5
\end{tabular}

number of photons enter the PSC in this spectral range. Having very small sizes (GQD-EDA: 1.8-3.5 $\mathrm{nm}$ and GQD-G: 3.5-6.5 $\mathrm{nm}$ ), GQDs will not show considerable light scattering effect. That is why the small negative change in $J_{\mathrm{SC}}$ in $400-800 \mathrm{~nm}$ spectral range does not change by changing the GQD concentration. However, the $\Delta J_{\mathrm{SC}}(\%)$ values in $300-400 \mathrm{~nm}$ spectral range increase by increasing the concentration of GQD-EDA in the LDS layer. This value attains $414 \%$ when the concentration is $1 \mathrm{M}$. When using very low concentrations of GQD-EDA in the LDS layer $(C<10 \mathrm{mM})$, light absorption and emission by GQDs are not enough to cause a significant change in IPCE and thereby in $J_{\mathrm{SC}}$.

Although there are reports in which bare PSC with high IPCE in $300-400 \mathrm{~nm}$ spectral range is fabricated, ${ }^{37}$ the LDS strategy owns a superiority in this regard. Application of the LDS layer on PSC not only increases the IPCE up to its highest attainable value but also improves the stability of the PSC by hindering the UV photons from reaching the PSC. The results exhibited in Fig. 3 and Table 1 imply that by embedding an adequate concentration of GQD-EDA in a thin LDS layer, a unity light harvesting efficiency could be attained in short wavelength region for PSC. It is worth mentioning that since the size of GQD-EDA particles are below $5 \mathrm{~nm},{ }^{25}$ even when using in high concentrations in the LDS layer, the light scattering by these particles will not be significant. The only problem which may limit the applied concentration of GQDS in the LDS layer is practical difficulties such as agglomeration of particles. Moreover, the interplay between GQD concentration and other parameters like RO and PA also affects the LDS layer performance. This will be discussed in detail in the next section.

\section{Effect of radiative overlap and parasitic absorption}

Quantum dots usually show a large overlap between their absorption and emission bands. ${ }^{38}$ But for GQDs this overlap can be very small or negligible. ${ }^{25}$ When there is an overlap between the absorption and emission bands of the luminescent material in the LDS layer, there is a probability that the emitted photons are re-absorbed by the luminescent material and a fraction of these photons will never get a chance to enter the solar cell depending on the QE. Therefore, RO affects the collection probability of down shifted photons (9) negatively and should be minimized. PA also has a great impact on the performance of the LDS layer. Although PA does not appear explicitly in the equations of the optical model, it affects the IPCE $\mathrm{IPCE}_{\mathrm{d}}$ is the IPCE associated with the photons that belongs to the PSC absorption band. If there is a large overlap between the absorption bands of the LDS layer and PSC, a considerable fraction of photons, which could be effectively converted into current by PSC, would be absorbed by the LDS layer before reaching the PSC. These photons might never get a chance to contribute in current generation because they meet several loss channels such as non-unity QE, RO, reflection loss etc. Therefore, the considerable decrease in $\mathrm{IPCE}_{\mathrm{d}}$ due to PA will not be compensated and total IPCE will be decreased.

In order to show the effect of RO and PA on the down shifting effect of GQDs in the LDS layer, the IPCE is calculated for PSC with LDS layer containing GQD-G particles. The absorption and 

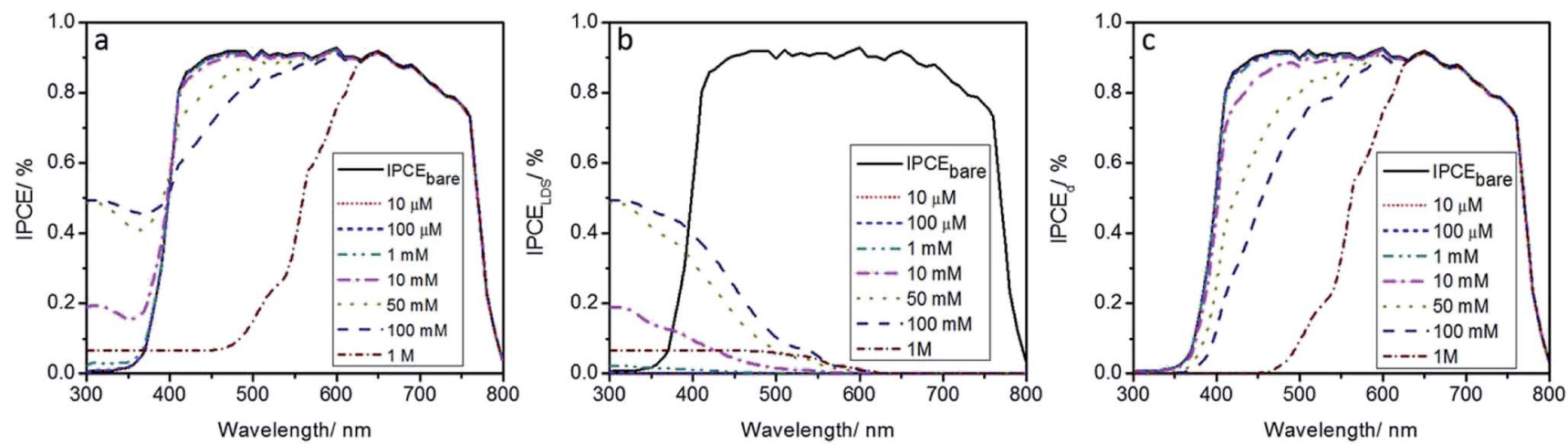

Fig. 4 Calculated (a) total IPCE spectra, (b) IPCE $E_{L D S}$ and (c) IPCE for $_{d}$ fSCs without and with LDS layers containing GQD-G with different concentrations.

emission spectra of GQD-G, exhibited in Fig. 2, show considerable RO and PA compared to that of GQD-EDA.

Fig. 4a shows the calculated IPCE for PSCs with LDS layers containing different concentrations of GQD-G. The thickness of LDS layer is $1 \mu \mathrm{m}$ and it is supposed that QE of GQD-G particles is the same as QE of GQD-EDA, i.e., 94\%. By increasing the concentration of GQD-G particles in the LDS layer from $10 \mu \mathrm{M}$ to $1 \mathrm{M}$, the IPCE of the PSC in $300-400 \mathrm{~nm}$ spectral range first increase and then decrease. Better down shifting effect in 300-400 $\mathrm{nm}$ spectral range attained for 10$100 \mathrm{mM}$ concentrations. Embedding higher concentrations of GQD-G in the LDS layer leads to a considerable decrease in calculated IPCE in $300-400 \mathrm{~nm}$ spectral range. This is attributed to the RO between the absorption and emission bands of GQD-G. Although at presence of higher concentration of GQD$G$, the number of down shifted photons increases, a large fraction of these photons is re-absorbed by GQD-G particles instead of entering the PSC. Eqn (10) shows that RO increases with concentration. The effect of RO on down shifting effect of GQD-G particles is much easily understood from Fig. 4b in which the calculated IPCE $_{\mathrm{LDS}}$ is shown for PSCs with LDS layers containing different concentrations of GQD-G. IPCE $_{\mathrm{LDS}}$ extends to around $600 \mathrm{~nm}$ because the down shifting of photons happens in all the absorption band of GQD-G. In the whole absorption range of the GQD-G (i.e., 300-600 nm), $\mathrm{IPCE}_{\mathrm{LDS}}$ increase by application of GQD-G up to $100 \mathrm{mM}$ and then decreases because of large RO in large concentrations. Although the IPCE $\mathrm{LDS}_{\mathrm{LD}}$ for $100 \mathrm{mM}$ concentration is larger than IPCE $_{\text {LDS }}$ for $50 \mathrm{mM}$ concentration in $400-600 \mathrm{~nm}$ spectral range (Fig. 4b), Fig. 4a shows a smaller total IPCE in this range for $100 \mathrm{mM}$ concentration compared to the IPCE for $50 \mathrm{mM}$ concentration. The reason lies in the fact that PA of photons in 400-600 $\mathrm{nm}$ spectral range in the LDS layer causes a substantial decrease in $\mathrm{IPCE}_{\mathrm{d}}$ in this spectral range (Fig. 4c). Since the absorption band of GQD-G (Fig. 2) extends to around $600 \mathrm{~nm}$, a large fraction of incident photons in $370-600 \mathrm{~nm}$ spectral range are absorbed in the LDS layer before reaching the PSC. These photons could be converted to current with IPCE around $90 \%$ if absorbed by PSC. But, being absorbed in the LDS layer, the probability of contribution of these photons in current generation is affected by different loss channels; RO being the
Table 2 Relative increase/\% in short-circuit current density of the PSCs with LDS layers containing different concentrations of GQD-G with respect to that of the reference cell for two wavelength ranges. The quantities are calculated from the corresponding IPCE spectra. The thickness of the LDS layers is $1 \mu \mathrm{m}$

\begin{tabular}{lcc}
\hline & $\Delta J_{\mathrm{SC}} / \%$ & \\
\cline { 2 - 3 } GQD-G concentration & $300-400 \mathrm{~nm}$ & $400-800 \mathrm{~nm}$ \\
\hline $10 \mu \mathrm{M}$ & -0.5 & -0.5 \\
$100 \mu \mathrm{M}$ & 0.3 & -0.5 \\
$1 \mathrm{mM}$ & 8.4 & -0.5 \\
$10 \mathrm{mM}$ & 78.6 & -0.8 \\
$50 \mathrm{mM}$ & 224.4 & -2.5 \\
$100 \mathrm{mM}$ & 239.0 & -5.3 \\
$1 \mathrm{M}$ & -53.1 & -35.1
\end{tabular}

most important one. This results in a negative effect of the LDS layer on the total IPCE of the PSC in 400-600 nm spectral range compared to the IPCE of bare PSC. Such decrease does not happen by using GQD-EDA because the RO and PA are negligible for it.

The relative change in the $J_{\mathrm{SC}}$ of the PSCs with GQD-G-filled LDS layers is calculated from the corresponding IPCE spectra of Fig. 4 for two spectral range, $300-400 \mathrm{~nm}$ and $400-800 \mathrm{~nm}$. The $\Delta J_{\mathrm{sC}}(\%)$ values are reported in Table 2. By increasing the concentration of GQD-G particles in the LDS layer, the $J_{\mathrm{SC}}$ associated with the absorption of photons in 300-400 $\mathrm{nm}$ spectral range increases up to $239 \%$ corresponding to $100 \mathrm{mM}$ concentration due to the down shifting effect of these particles. A sudden decrease in $J_{\mathrm{SC}}$ then is observed because of the large RO loss. In 400-800 nm, PA causes a negative $\Delta J_{\mathrm{SC}}$ in all concentrations.

Similar behavior in IPCE and $J_{\mathrm{SC}}$ is observed when using GQD-G LDS layers with different thicknesses on PSC. Additionally, the escape loss from side planes become notable for thick LDS layers. The calculated IPCE spectra for the PSCs with GQD-G LDS layers with different thicknesses are provided in Fig. S2 in ESI. $\dagger$

\section{Effect of luminescent quantum efficiency}

Luminescent $\mathrm{QE}$ is the ratio of the number of emitted photons to the number of absorbed photons by the luminescent 
particles. In order to have an efficient LDS layer, the embedded luminescent particles should have a large QE. GQDs with QEs greater than $90 \%$ are synthesized by simple methods. ${ }^{25}$ In this regard, GQDs can be suitable candidates for application in LDS layers. In previous sections, the application of GQD-EDA LDS layer on PSC was investigated by calculation of the IPCE of PSCs with this layer. Similar calculation with the LDS layers containing GQD-G particles is also provided. Although the actual QE of GQD-EDA and GQD-G particles are reported to be $94 \%$ and $6 \%$ in, ${ }^{25}$ in calculations of previous sections the QE is taken as $94 \%$, so that the effect of RO and PA could be investigated independent of the QE. But in practice, QE determines the amount of negative effect of RO and PA on the performance of the LDS layer. When QE is small, the re-absorbed photons by GQD or the photons in the parasitic absorption range, have a small chance to be emitted by GQDs again, reach the PSC and contribute in current generation. Therefore, the amount of loss due to the RO or PA is considerable. When QE is large, the absorbed photons by GQDs are re-emitted by large probability. Therefore, the larger the QE, the smaller the RO and PA loss. However, the probability that the re-emitted photons reach the PSC is defined by other optical loss channels such as reflection at interfaces or escape cone loss.

The effect of QE can be observed in Fig. 5 in which the calculated IPCE is shown for two PSCs with LDS layers containing $50 \mathrm{mM}$ GQD-G with $94 \%$ and 6\% QE compared to the IPCE of a bare PSC. Although the amount of RO and PA is the same in both LDS layers, larger QE led to a larger IPCE in both 300-400 $\mathrm{nm}$ and 400-600 $\mathrm{nm}$ spectral range. The amount of increase in IPCE in 300-400 $\mathrm{nm}$ spectral range is considerably larger when using GQDs with $94 \% \mathrm{QE}$, because a larger fraction of absorbed photons by GQD-G particles in this range are down shifted and contribute in current generation in PSC. In 400$600 \mathrm{~nm}$ spectral range, the $\mathrm{IPCE}_{\mathrm{d}}$ and thereby total IPCE decreases mainly due to PA. However, larger QE (94\%) helps to downgrade the amount of reduction in IPCE in 400-600 spectral range. In fact, the amount of loss due to the PA and RO is not defined just by the RO and PA parameters and is affected by the

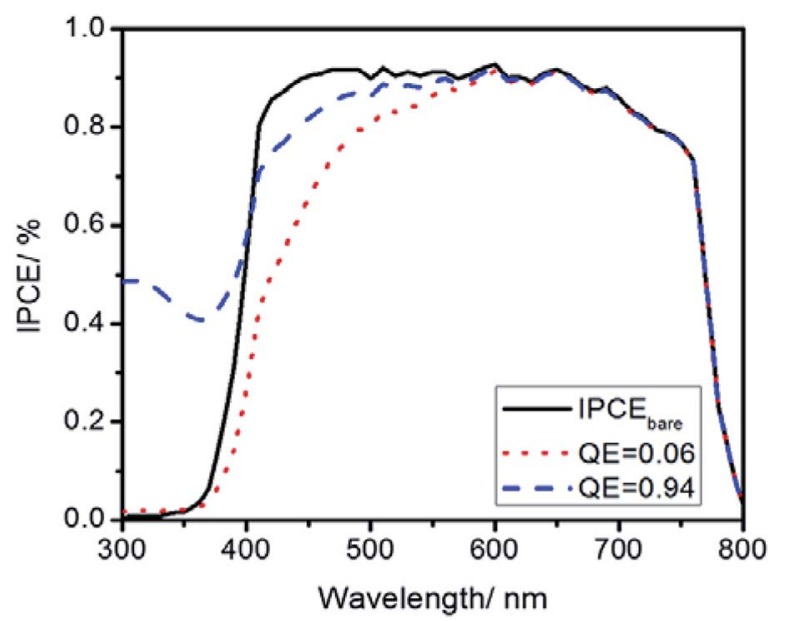

Fig. 5 Calculated IPCE for PSCs without and with LDS layers containing GQD-G with different QEs (6\% and 94\%).
QE. It is worth mentioning that even unity QE does not fully compensate for RO and PA losses. This can be perceived by comparing the results depicted in Fig. 3 and 4. Although the QE is set to be $94 \%$ in both related calculations, larger RO and PA for GQD-G compared to GQD-EDA led to smaller IPCEs for PSCs coupled to LDS layers containing these GQD-G.

\section{Conclusions}

Better use of the solar spectrum along with improved stability of PSC is possible by inclusion of an LDS layer on PSC. Although UV photons can be absorbed and converted to current in PSC, the stability of perovskite material is highly affected by these high energy photons. Down shifting UV photons to lower energy photons in visible region, gives the opportunity for both preventing high energy photons from reaching the perovskite absorber inside the PSC and their contribution in current generation. However, the most beneficial effects will be accomplished if losses associated with RO, PA and low quantum efficiency are avoided, i.e., the quantum efficiency is as high as possible (unity preferred) and the overlap between the absorption and emission bands of down shifting material and also the overlap between its absorption band and PSC spectral response in visible region is minimized. Here we demonstrated that GQDs, showing absorption and emission in UV and visible region respectively, are suitable candidates for using in LDS layers for PSCs. There are various reports on synthesis of GQDs with large QE and very small RO. Moreover, GQD with least overlap of its absorption band with visible region can be synthesized; this provides low PA. Our calculation based on a simple optical model showed that application of LDS layer containing GQDs on the PSC causes a significant enhancement in IPCE of the PSC. By using a proper GQD with $94 \%$ QE and negligible RO and PA, a remarkable increase of more than $400 \%$ in short circuit current density corresponding to the light absorption in 300-400 nm spectral range is achievable. The results of optical modeling are used for an optimum design of an LDS layer that contains GQD as down shifting material to improve stability and efficiency of PSC. Experimental verification of the above results is in progress.

\section{Conflicts of interest}

There are no conflicts to declare.

\section{References}

1 D. M. Jang, K. Park, D. H. Kim, J. Park, F. Shojaei, H. S. Kang, J.-P. Ahn, J. W. Lee and J. K. Song, Nano Lett., 2015, 15, 51915199.

2 S. Kazim, M. K. Nazeeruddin, M. Grätzel and S. Ahmad, Angew. Chem., Int. Ed., 2014, 53, 2812-2824.

3 M. L. Petrus, J. Schlipf, C. Li, T. P. Gujar, N. Giesbrecht, P. Müller-Buschbaum, M. Thelakkat, T. Bein, S. Hüttner and P. Docampo, Adv. Energy Mater., 2017, 7, 1700264.

4 M. M. Lee, J. Teuscher, T. Miyasaka, T. N. Murakami and H. J. Snaith, Science, 2012, 338, 643-647. 
5 M. A. Green, Y. Hishikawa, E. D. Dunlop, D. H. Levi, J. HohlEbinger and A. W. Y. Ho-Baillie, Prog. Photovoltaics Res. Appl., 2018, 26, 3-12.

6 T. Leijtens, G. E. Eperon, S. Pathak, A. Abate, M. M. Lee and H. J. Snaith, Nat. Commun., 2013, 4, 1-8.

7 A. Gheno, T. Trigaud, J. Bouclé, P. Audebert, B. Ratier and S. Vedraine, Opt. Mater., 2018, 75, 781-786.

8 H. Zhang, H. Wang, H. Zhu, C. Chueh, W. Chen, S. Yang and A. K.-Y. Jen, Adv. Energy Mater., 2018, 8, 1702762.

9 F. Lang, O. Shargaieva, V. V. Brus, H. C. Neitzert, J. Rappich and N. H. Nickel, Adv. Mater., 2017, 30, 1702905.

10 N. H. Nickel, F. Lang, V. V. Brus, O. Shargaieva and J. Rappich, Adv. Electron. Mater., 2017, 3, 1700158.

11 R. Ruess, F. Benfer, F. Böcher, M. Stumpp and D. Schlettwein, ChemPhysChem, 2016, 17, 1505-1511.

12 G. Niu, W. Li, F. Meng, L. Wang, H. Dong and Y. Qiu, J. Mater. Chem. A, 2014, 2, 705-710.

13 J.-P. Correa-Baena, M. Saliba, T. Buonassisi, M. Grätzel, A. Abate, W. Tress and A. Hagfeldt, Science, 2017, 358, 739744.

14 Q. Luo, H. Chen, Y. Lin, H. Du, Q. Hou, F. Hao, N. Wang, Z. Guo and J. Huang, Adv. Funct. Mater., 2017, 27, 1702090.

15 Z. Hosseini, N. Taghavinia and E. W.-G. Diau, ChemPhysChem, 2017, 18, 3292-3308.

16 T. F. Schulze and T. W. Schmidt, Energy Environ. Sci., 2015, 8, 103-125.

17 Y. Jestin in Comprehensive Renewable Energy, ed. A. Sayigh, Elsevier, 2012, vol. 1, ch. 1.26, pp. 563-85.

18 E. Klampaftis, D. Ross, K. R. McIntosh and B. S. Richards, Sol. Energy Mater. Sol. Cells, 2009, 93, 1182-1194.

19 Z. Hosseini, W.-K. Huang, C.-M. Tsai, T.-M. Chen, N. Taghavinia and E. W.-G. Diau, ACS Appl. Mater. Interfaces, 2013, 5, 5397-5402.

20 Z. Hosseini, N. Taghavinia and E. W.-G. Diau, Mater. Lett., 2017, 188, 92-94.

21 F. Bella, G. Griffini, J.-P. Correa-Baena, G. Saracco, M. Gratzel, A. Hagfeldt, S. Turri and C. Gerbaldi, Science, 2016, 354, 203-206.
22 N. Chander, A. F. Khan, P. S. Chandrasekhar, E. Thouti, S. K. Swami, V. Dutta and V. K. Komarala, Appl. Phys. Lett., 2014, 105, 33904.

23 Z. Hosseini, E. W. G. Diau, K. Mehrany and N. Taghavinia, ChemPhysChem, 2014, 15, 3791-3799.

24 M. L. Mueller, X. Yan, J. A. McGuire and L. Li, Nano Lett., 2010, 10, 2679-2682.

25 D. Qu, M. Zheng, L. Zhang, H. Zhao, Z. Xie, X. Jing, R. E. Haddad, H. Fan and Z. Sun, Sci. Rep., 2014, 4, 5294.

26 X. Yan, X. Cui and L. Li, J. Am. Chem. Soc., 2010, 132, 59445945.

27 Z. Gan, H. Xu and Y. Hao, Nanoscale, 2016, 8, 7794-7807.

28 T. Majumder and S. P. Mondal, J. Electroanal. Chem., 2016, 769, 48-52.

29 S. Zhuo, M. Shao and S.-T. Lee, ACS Nano, 2012, 6, 10591064.

30 S. Chandra, P. Patra, S. H. Pathan, S. Roy, S. Mitra, A. Layek, R. Bhar, P. Pramanik and A. Goswami, J. Mater. Chem. B, 2013, 1, 2375.

31 S.-H. Choi, J. Phys. D: Appl. Phys., 2017, 50, 103002.

32 L. Li, G. Wu, G. Yang, J. Peng, J. Zhao and J.-J. Zhu, Nanoscale, 2013, 5, 4015.

33 D. Alonso-Álvarez, D. Ross, E. Klampaftis, K. R. McIntosh, S. Jia, P. Storiz, T. Stolz and B. S. Richards, Prog. Photovoltaics Res. Appl., 2015, 23, 479-497.

34 B. Hong, Sol. Energy Mater. Sol. Cells, 2003, 80, 417-432.

35 D. Alonso-álvarez, D. Ross and B. S. Richards, Proceeding Photovolt. Spec. Conf., 2012, pp. 9-14.

36 L. Tang, R. Ji, X. Cao, J. Lin, H. Jiang, X. Li and K. S. Teng, ACS Nano, 2012, 6, 5102-5110.

37 H. S. Anizelli, V. Stoichkov, R. V. Fernandes, J. L. Duarte, E. Laureto, J. Kettle, I. Visoly-Fisher and E. A. Katz, Organic Electronics: physics, materials, applications, 2017, 49, 129134.

38 E. Klampaftis, D. Ross, K. R. McIntosh and B. S. Richards, Sol. Energy Mater. Sol. Cells, 2009, 93, 1182-1194. 